\title{
Focal Shifting of Double Ring Shaped Radially Polarized Multi Gaussian Beam
}

\author{
K.Prabakaran ${ }^{1 *}$, K.B.Rajesh², V.Hariharan'1, A. Mohamed Musthafa ${ }^{3}$, \\ M.Velu' ${ }^{1}$, V.Aroulmoji ${ }^{4}$ \\ ${ }_{1}^{1}$ Department of Physics, Mahendra Arts and Science College (Autonomous), Namakkal, Tamilnadu, India \\ ${ }^{2}$ Department of Physics, Chikkanna Government Arts College, Trippur, Tamilnadu, India \\ ${ }^{3}$ Department of General Studies (Physics Group), Jubail University College (Male Branch), \\ Royal Commission of Jubail, Kingdom of Saudi Arabia \\ ${ }^{4}$ Center for Research and Development, Mahendra Engineering College (Autonomous), \\ Mallasamudram, 637 503, Tamil Nadu, India
}

\begin{abstract}
Tight focusing of double ring shaped radially polarized multi gaussian beam with cosine phase plate is studied numerically based on vector diffraction theory. For higher-order, radially polarized mode beams as well as a fundamental mode $\left(\mathrm{R}-\mathrm{TEM}_{01}{ }^{*}\right)$ beam, the strong longitudinal component forms a sharper spot at the focal point under a high-NA focusing condition. In particular, double-ring-shaped radially polarized mode (R-TEM $11^{*}$ ) beams can effectively reduce the focal spot size because of destructive interference between the inner and the outer rings. The mathematical expressions for the focused fields are derived. Simulation results show that the focused fields and phase distributions at focus are largely influenced by both the cosine parameter and beam diameter of the incident beams. Moreover, focal spot with flat-topped, two focal spots patterns can be flexibly achieved by carefully choosing the cosine parameter $(\mathrm{C})$ and the ratios of the pupil diameter to the beam diameter $(\beta)$ which confirms the potential of such beams in wide applications, such as optical tweezers, lithography, and material processing.
\end{abstract}

KEYWORDS: Vector diffraction theory, High NA Lens, Multi Gaussian beam, Optical trapping.

\section{INTRODUCTION}

Tight focusing of radially polarized light is a very topical area of research [1-3]. It has been shown that, in many circumstances, focusing of radially polarized light results in a smaller focal spot than focusing linearly polarized light. Applications are in microscopy, lithography, metrology, nonlinear optics, optical manipulation, and optical micromachining. Gaussian beams are very similar to researchers and have found wide applications in many optical systems; however, this kind of beam is only one solution of the Helmholtz equation. In fact, Gaussian beam is one special case of Hermite-sinusoidal-Gaussian beams that are more general solution and were introduced by Casperson et.al. [4-6]. Hyperolic-cosine-Gaussian (cosh-Gaussian) beams are regarded as the special case of Hermitesinusoidal Gaussian beams, and Gaussian beams are still base cases of cosh-Gaussian beams. The coshGaussian beams have attracted much attention because their profiles can be altered by choosing the suitable decentered parameters in cosh parts [7-9], and propagation and focusing properties of cosh-Gaussian beams have become object of many works [10]. Zhang et al proposed the virtual sources for a cosh-Gaussian beam [11]. Zhang et al proposed the virtual sources for a multi Gaussian beam [12]. However, most of the studies on the radially polarized beam and its applications have concerned the so-called doughnutshaped beam, which is represented as $\left(\mathrm{TM}_{01}\right.$ mode)
$[13,14]$. Recently, Kozawa et al report that the radially polarized higher-order Laguerre-Gaussian beam (LG) has higher a fundamental radially polarized mode performance than the low-order one and can generate the focal spot beyond the Abbe's diffraction limit $0.5 \lambda$. This kind of higher-order LG beam has concentric multi ring-shaped intensity pattern with $\pi$ phase shift between the rings[15-17].

In the optical trapping system, it is usually deemed that the forces exerted on the particles in light field include two kinds of forces; one is the gradient force, which is proportional to the intensity gradient, and the other is the scattering force, which is proportional to the optical intensity $[18,19]$. Therefore, the tunable focal shift predicts that the position of optical trap may be controllable. In fact, tracing the movement of the point of absolute maximum intensity along the axis has become the subject of a number of publications for several decades [20-23]. It was found that the point of absolute maximum intensity does not coincide with the geometrical focus but shifts along the axis towards the aperture of the optical system [22-24]. More interestingly, the focal shift may be in continuous in certain optical focusing systems. It was found that the focal shift may be accompanied by an effective permutation of the focal point, and this effect is referred to as focal switch $[25,26]$. Focal shifts have taken an important part in design and optimization of various optical systems and extremely important for

*Corresponding Author: prabakaran27mar@gmail.com

Received: $15.06 .2019 \quad$ Accepted: 18.07.2019

Published on: 28.08.2019 
image formation in defocused planes, for increasing the depth of focus and in automatic focusing [27]. More interestingly, the focal shift may be in continuous in certain optical focusing systems. It was found that the focal shift may be accompanied by an effective permutation of the focal point, and this effect is referred to as focal switch $[28,29]$. In our knowledge, the focusing properties of the double ring shaped radially polarized multi gaussian beam with radial cosine phase plate are not studied. In this paper the focal shift of the double ring shaped radially polarized multi gaussian beam with radial cosine phase plate is investigated theoretically by vector diffraction theory.

\section{VECTOR DIFFRACTION THEORY OF DOUBLE RING SHAPED RADIALLY POLARIZED MULTI GAUSSIAN BEAM}

A schematic configuration of the proposed method is shown in Fig.1. An incident double ring shaped radially polarized multi gaussian beam transmits through a radial cosine phase plate and is subsequently focused by a high NA lens. The analysis was performed on the basis of Richards and Wolf's vectorial diffraction method [30] widely used for high-NA lens system at arbitrary incident polarization. In the case of the incident polarization, adopting the cylindrical coordinates $r, z, \phi$ and the notations of Ref. [31], the electric field E(r,z, $\phi)$ in the vicinity of the focal region can be written as

$$
\vec{E}(r, z)=E_{r} \vec{e}_{r}+E_{z} \vec{e}_{z} \rightarrow(1)
$$

Where $E_{r}$, and $E_{z}$ are the amplitudes of the two orthogonal components and $\vec{e}_{r}$ and $\vec{e}_{z}$ are their corresponding unit vectors. The two orthogonal components of the electric field is given as

$$
\begin{aligned}
& E_{r}(r, z)=A \cos \phi \int_{0}^{\theta \max } \cos ^{1 / 2}(\theta) T(\theta) P(\theta) \sin 2 \theta J_{1}(k r \sin \theta) e^{i k z \cos \theta} d \theta \rightarrow(2) \\
& E_{z}(r, z)=2 i A \cos \phi \int_{0}^{\theta \max } \cos ^{1 / 2}(\theta) T(\theta) P(\theta) \sin ^{2} \theta J_{0}(k r \sin \theta) e^{i k z \cos \theta} d \theta \rightarrow(3)
\end{aligned}
$$

Where $\alpha=\arcsin (N A) / n$ the maximal angle is determined by the numerical aperture of the objective lens, and $\mathrm{n}$ is the index of refraction between the lens and the sample. $k=2 \pi / \lambda$ is the wave number and $\mathrm{Jn}_{\mathrm{n}}(\mathrm{x})$ is the Bessel function of the first kind with order $n . r$ and $z$ are the radial and $\mathrm{z}$ coordinates of observation point in focal region, respectively. $\varphi$ describes the pupil apodization function. The intensity in the focal region is proportional to the square modulus of Eq. (1). In the system investigated in this chapter, the wavefront phase distribution is radial cosine function distribution, and can be written as [32]

$$
\varphi=\pi \cdot \cos \left[\pi \cdot \mathrm{C} \frac{\tan (\theta)}{\tan (\alpha)}\right] \rightarrow(4)
$$

where $\mathrm{C}$ is the frequency parameter in cosine part of the wavefront phase distribution, $\mathrm{C}$ denotes the radial change frequency of the phase and parameter. The reason for choosing this kind of radial cosine phase wavefront is that it is very simple and easy to carry out, for example this kind of phase distribution can be implemented by phase spatial light modulator or by pure phase plate manufactured by the lithographic method conveniently. The electric field in the focal region can be written in the same form as Eq. (1); however, the three orthogonal components Er, and Ez are different and should be expressed as

$$
\begin{aligned}
& E_{r}(r, z)=A \int_{0}^{\theta \max } \cos ^{1 / 2}(\theta) T(\theta) \times P(\theta) \times \pi \cdot \cos \left[\pi \cdot C \frac{\tan (\theta)}{\tan (\alpha)}\right] \sin 2 \theta J_{1}(k r \sin \theta) e^{i k z \cos \theta} d \theta \rightarrow(5) \\
& E_{z}(r, z)=2 i A \int_{0}^{\theta \max } \cos ^{1 / 2}(\theta) T(\theta) \times P(\theta) \times \pi \cdot \cos \left[\pi \cdot C \frac{\tan (\theta)}{\tan (\alpha)}\right] \sin ^{2} \theta J_{0}(k r \sin \theta) e^{i k z \cos \theta} d \theta \rightarrow(6)
\end{aligned}
$$

The total intensity distribution in the focal region is proportional to the square modulus of Eq. (1), the radial polarized component and longitudinal polarized component; azimuthally polarized component can be calculated according to Eqs. (5) and (6) respectively. The $T(\theta)$ describes the double ring shaped radially polarized multi gaussian beam, this function is given by [12]

$$
P(\theta)=\left(\frac{\theta}{\theta_{0}}\right)^{m} \sum_{n=-N}^{N} \exp \left[-\left(\frac{\theta-\theta_{c}-n \omega_{0}}{\omega_{0}}\right)^{2}\right] \rightarrow(7)
$$


Here, $\theta$ is the converging semi-angle. We denote the maximum converging semi-angle as $\theta_{\max }$ which is related to objective numerical aperture by $\theta_{\max }=\arcsin (N A)$. $\theta_{0}$ is an angle which, along with integer $m$, determines the shape of the modulation function. $\theta_{0}$ is usually chosen to be slightly smaller than $\theta_{\max } \theta_{c}$ determines the radial position translation of $P(\theta)$. Here we take $\theta_{c}=\theta_{\max } / 2$. $w_{0}$ is the waist width of single Gaussian beam which is calculated by the following formula

$$
w_{0}=1 / 2 \times \frac{\theta \max }{N+\left\{1-\ln \left[\sum_{n=-N}^{N} \exp \left(-n^{2}\right)\right]\right\}^{1 / 2}} \rightarrow(8)
$$

Equation (7) describes an object beam. It is suitable to convert Eq. (8) from a function of angle into a function of radial polar coordinate. One may substitute $\theta$ with $\arcsin (r / f)$, where $f$ is the focal distance of the objective. In Eq. (7), the factor $\left(\theta / \theta_{0}\right)$ measures the most of light energy is located on the annular edge of the pupil. Increasing the integer $m$ concentrates more energy into the annular edge area in which the converging semiangle is more than $\theta_{0}$. The sum of $(2 N+1)$ spatially equally spaced Gaussian beams ensures that amplitude of the constructed annular multi-Gaussian beam decreases suddenly, when reaching the outer edge of the pupil. $\mathrm{T}(\theta)$ denotes the apodization function of the $\mathrm{LG}_{11}$ beam, which is given as [15-17],

$$
T(\theta)=\beta^{2} \frac{\sin \theta}{\sin ^{2} \theta} \exp \left[-\left(\beta \frac{\sin \theta}{\sin \theta_{\max }}\right)^{2}\right] L_{p}^{1}\left[2\left(\beta \frac{\sin \theta}{\sin \theta_{\max }}\right)^{2}\right] \rightarrow \text { (9) }
$$

Here $\mathrm{Lp}$ is the generalized Laguerre polynomial and $\beta$ is the ratio of the pupil radius to the incident beam radius in front of the focusing lens. Note that $\beta$ should be greater than 1 because the outer ring of the R-TEM ${ }_{11}$ beam will be completely truncated by the pupil if $\beta \leq 1$. In this study the NA is assumed to be 1.2 , with $n=1.33 . A$ is determined such that each truncated beam has the same optical power.

\section{RESULTS AND DISCUSSION}

Without loss of validity and generality, the focusing properties of the double ring shaped radially polarized multi Gaussian beam with radial cosine phase wave front are calculated. We perform the integration of Eq. (1) numerically using parameters $\lambda=1, \omega_{0}=0.125, \mathrm{NA}=0.9$ and $\mathrm{m}=1$. Here, for simplicity, we assume that the refractive index $n$ $=1$ and $A=1$. For all calculation in the length unit is normalized to $\lambda$ and the energy density is normalized to unity. In order to understand the focusing properties of the double ring shaped radially polarized multi Gaussian beam extensively, firstly, the focusing of double ring shaped radially polarized multi Gaussian beam with high NA lens system is investigated without wave front phase modulation. The intensity distribution of a radially polarized multi Gaussian incident beam for the high NA lens is shown in Fig.2 (a-c). It is observed from the Fig.2a shows the intensity profile of the radial, longitudinal and total electric field components of the optical field at focus. It is evident that the intensity of the longitudinal component (blue line) is higher than the radial component (red line). From the Fig.2a, we measured the FWHM of the generated focal spot size is $1.2 \lambda$ and focal depth $2 \lambda$ which is shown in Fig. $2 \mathrm{~b}$. Such a focal spot segment is useful for high refractive index particle trapping. The Fig. $3(\mathrm{a}, \mathrm{b} \& \mathrm{c})$ shows the focal segment generated for $\beta=1$, for cosine parameter values of $\mathrm{C}=0.25, \mathrm{C}=0.5$, and $\mathrm{C}=1$. It can be seen from the Fig.3 (a) shows that the generated focal segment is a shifted focal spot. From the Fig.3 (a) the maximum intensity position shifted to $-0.9 \lambda$, However when we increased cosine parameter (C) values for $\mathrm{C}=0.5$ and $\mathrm{C}=1$ the maximum intensity position shifted to $-1 \lambda$, and $-2 \lambda$ respectively is shown in fig.3 (b \& c). We observed that the generated focal spot in focal region shifts towards the optical aperture along optical axis on increasing cosine parameter $\mathrm{C}$, namely focal shift phenomenon occurs.Figure. 4 shows the intensity distributions of double ring shaped radially polarized multi gaussian beam without cosine plate for $\beta=1.26$. As shown in Fig.4(a), we measured FWHM of the splited focal spot is $0.8 \lambda$ and corresponding radial component percentage is $70 \%$ of the total intensity and the FWHM of the sub wavelength focal spot size is about 0.251 , and focal depth of $2.8 \lambda$ as shown in Fig. $4 b$, axially separated distance is $2.8 \lambda$ is shown in fig. 4 (c). Such type of focal spot used for multiple optical trapping applications. The Fig.5 (a, b \& c) shows the focal segment generated for $\beta=1.26$, for cosine parameter values of $\mathrm{C}=0.25, \mathrm{C}=0.5$, and $\mathrm{C}=1$. It can be seen from Fig.5 (a) shows that the generated focal segment is a two focal spots. From the Fig.5(a-c) we observed that the generated focal spots in focal region shifts towards the optical aperture along optical axis on increasing cosine parameter $\mathrm{C}$. However when we change the $\beta$ value from 1 to 1.26 the single focal spot is changed to two focal spots segment. The Fig.6 shows the focal structure generated by double ring shaped multi gaussian beam tightly focused with high NA lens. It is observed from Fig $6(\mathrm{a})$, when $\beta=1.08$ and the cosine parameter value $C=0$, the generated focal generated focal structure is a flattop profile having FWHM of $1.6 \lambda$ and focal depth of 3.8 $\lambda$. The 2D intensity calculated at the focus illustrates that the focal 
structure is dominated by the longitudinal component and the radial intensity remain as a intensity as $80 \%$ of the total intensity. Now, the beam parameter $\beta$ is chosen as 1.08 to investigate its effect on focal shift. The intensity distributions for $(\beta=1.08)$ and different $C$ are illustrated in Fig.7. It can be seen from this figure that the beam parameter $C$ affects focal intensity distribution very considerably. On increasing phase parameter $\mathrm{C}$, one focal peak evolves into one dark focus that is defined as those whose intensity is lower than that around it, namely, local focus intensity minimum. There is one optical intensity maximums along optical axis. On increasing phase parameter $\mathrm{C}$, whole focal pattern shifts along optical axis near from the optical aperture, one intensity peak shrinks so that there is only one optical intensity maximum. The dependence of focal shift on $\mathrm{C}$ is also given in Fig.7(a-c). If this focal peak is used to construct one optical trap, cosine parameter C may be employed to adjust trap position, in turn, can transport micro particles.

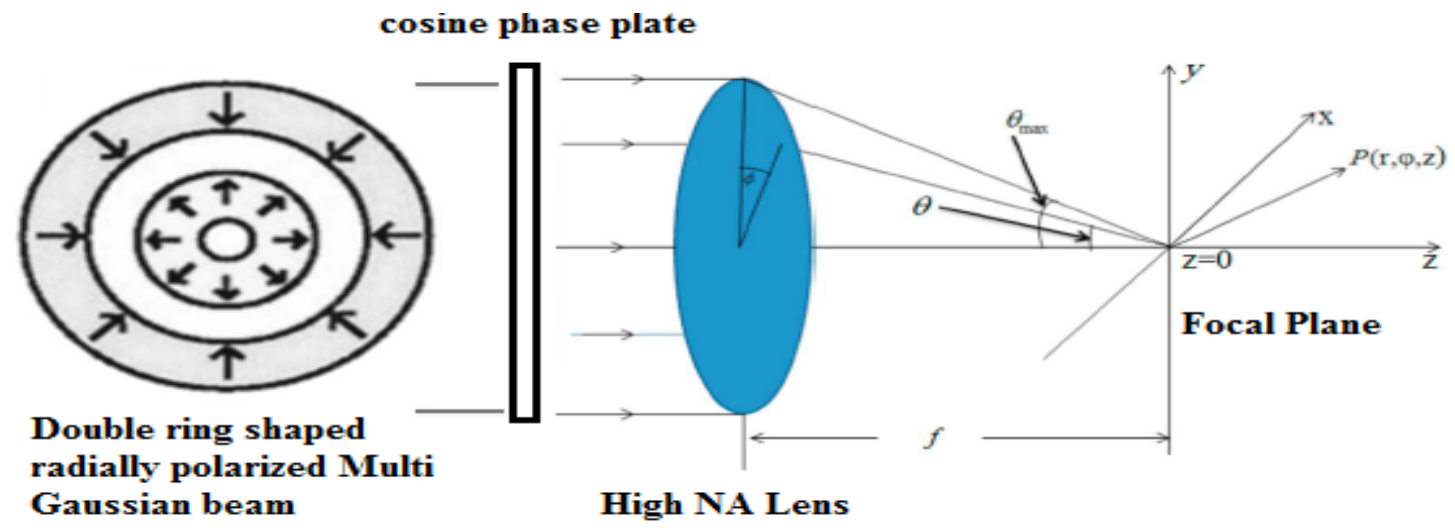

Fig.1. Focusing of a double ring shaped radially polarized multi Gaussian beam with radial cosine phase plate by high NA lens

(a)

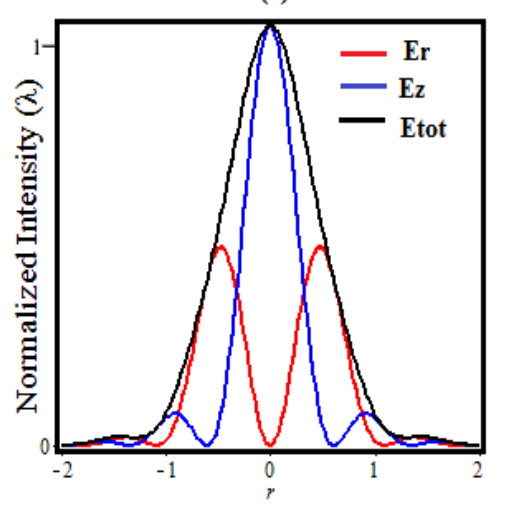

(b)

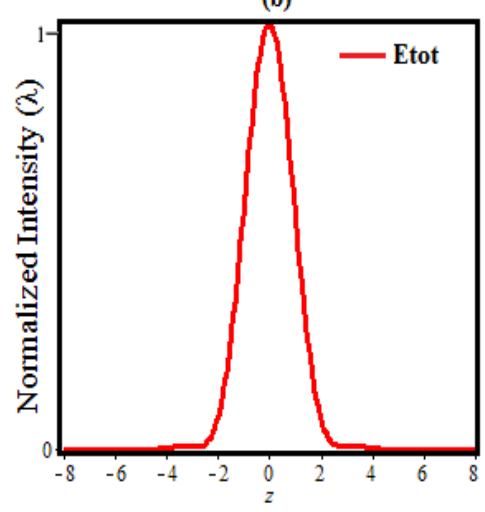

(c)

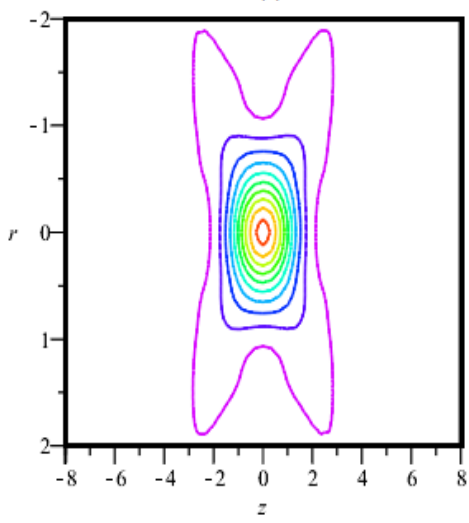

Fig.2. (a) 2D intensity distribution at $\mathrm{z}=0 \lambda$. (b) $0 \mathrm{n}$ axial intensity at $\mathrm{r}=0 \lambda$ (c) $3 \mathrm{D}$ intensity distributions in $r-z$ plane incident R-TEM $11^{*}$ beam for $\beta=1$ without wave front phase modulation.

(a)

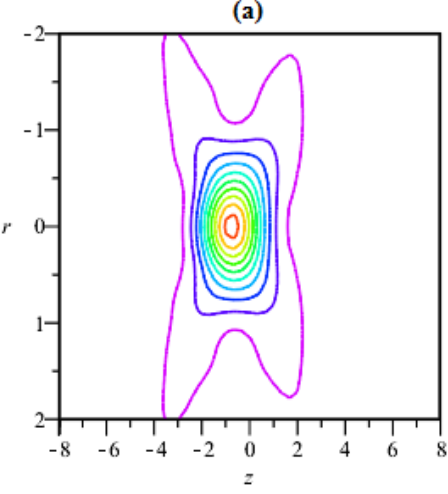

(b)

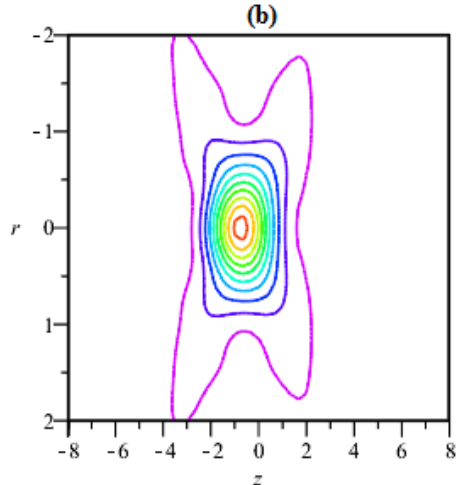

(c)

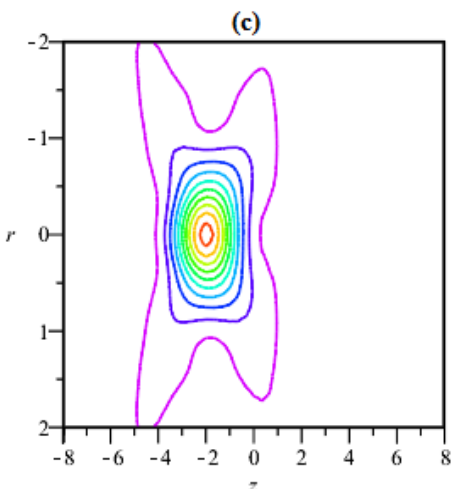

Fig.3. Normalized total intensity distributions in $\mathrm{r}-\mathrm{z}$ plane incident $\mathrm{R}-\mathrm{TEM}_{11}{ }^{*}$ beam under the condition of $\beta=1$, (a ) $C=0.25$, (b) $C=0.5$ (c ) $C=1$ respectively. 

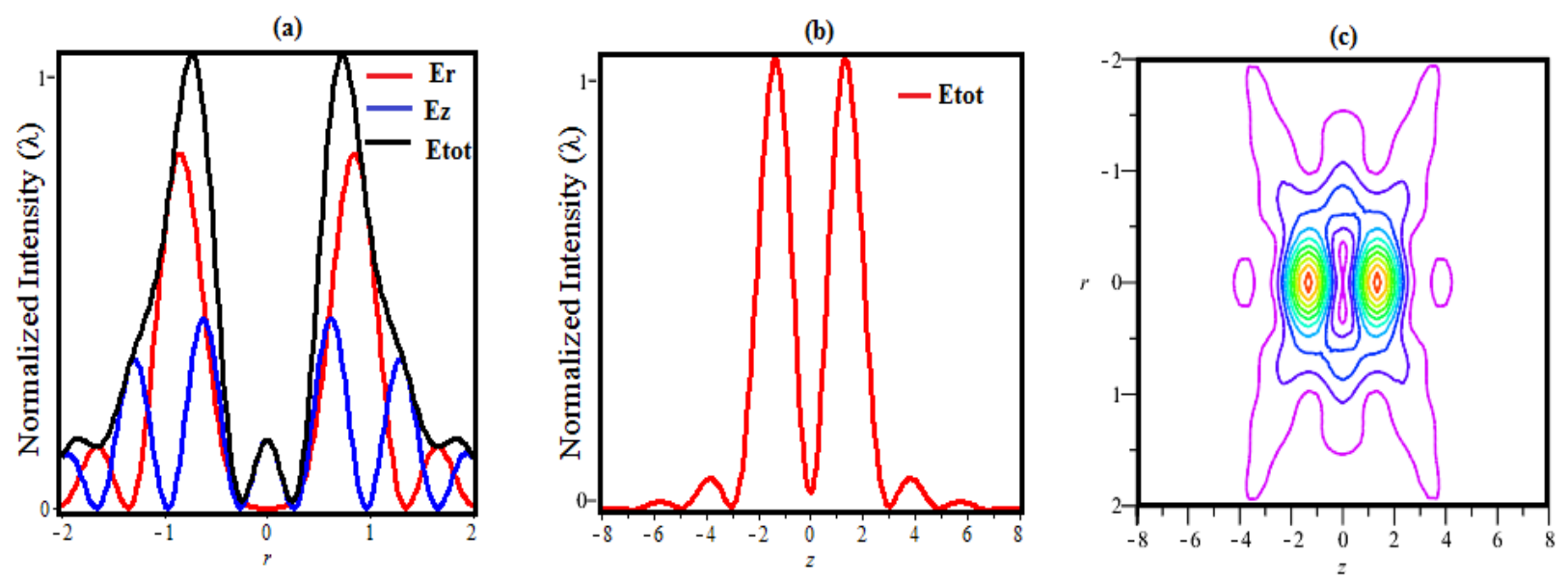

Fig.4. (a) 2D intensity distribution at $\mathrm{z}=0 \lambda$. (b) 0 axial intensity at $\mathrm{r}=0 \lambda$ (c) 3D intensity distributions in r-z plane incident R-TEM ${ }_{11}$ * beam under the condition of $\beta=1.26$ without wave front phase modulation.

(a)

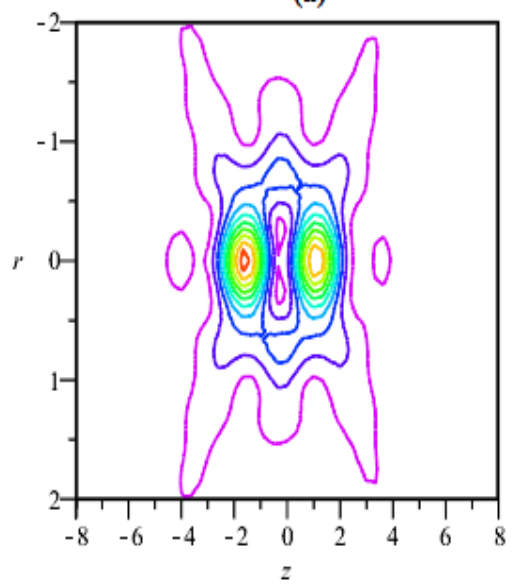

(b)

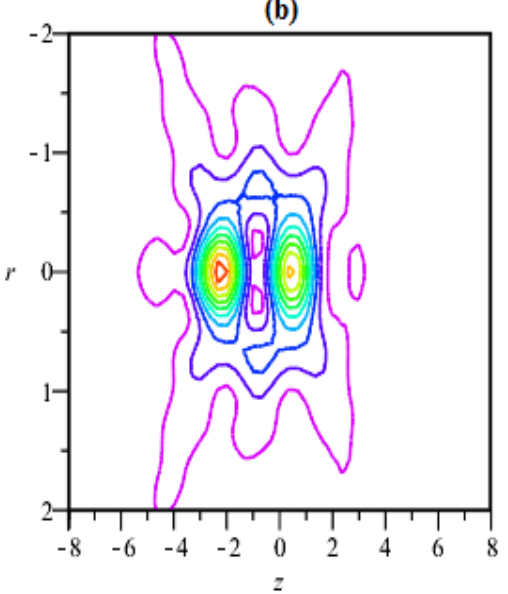

(c)

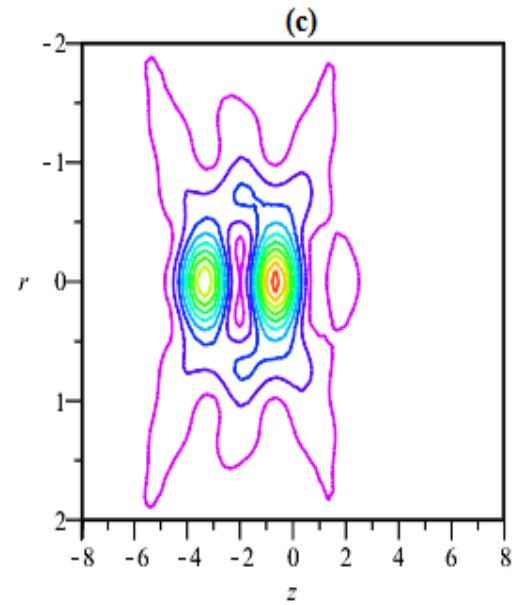

Fig.5. Normalized total intensity distributions in $r-z$ plane incident R-TEM ${ }_{11} *$ beam under the condition of $\beta=1.26$, (a) $C=0.25$, (b) $C=0.5$ (c) $C=1$ respectively.
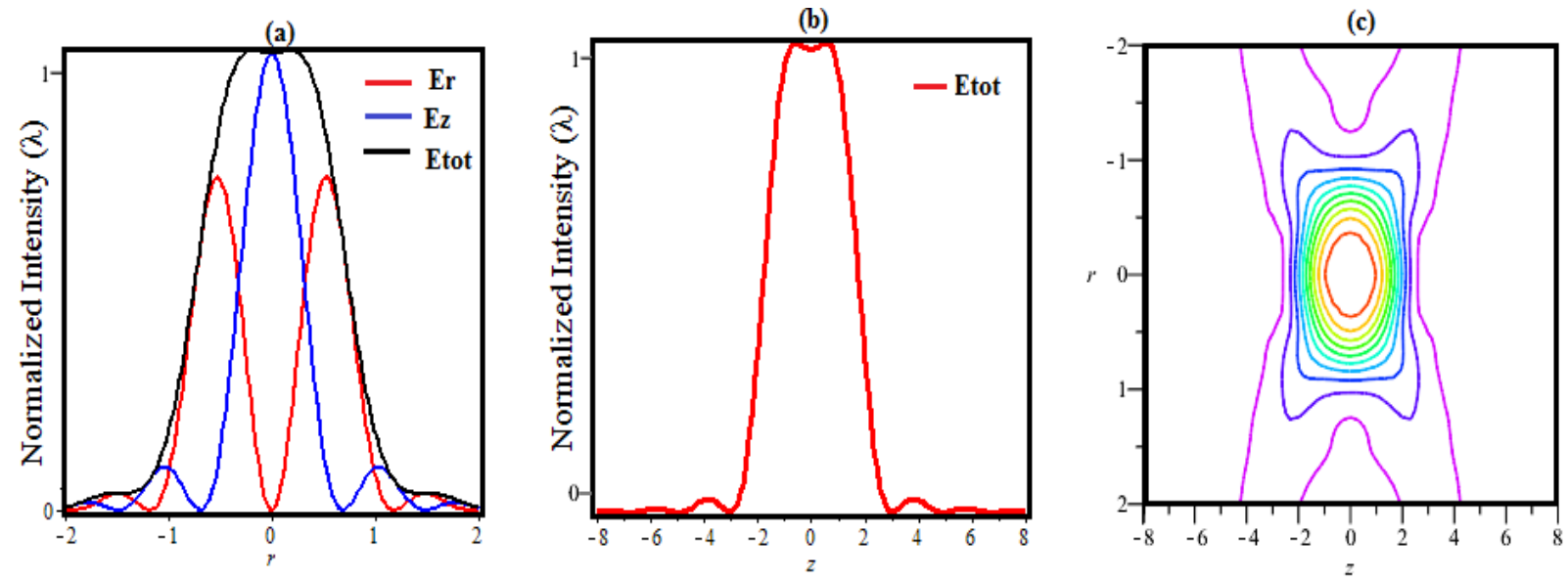

Fig.6. (a) 2D intensity distribution at $\mathrm{z}=0 \lambda$. (b) 0 axial intensity at $\mathrm{r}=0 \lambda$ (c) $3 \mathrm{D}$ intensity distributions in $r-z$ plane incident R-TEM 11 * beam under the condition of $\beta=1.08$ without wave front phase modulation. 
(a)

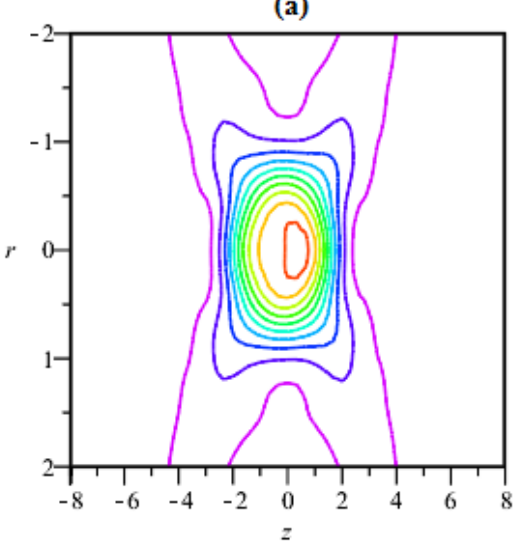

(b)

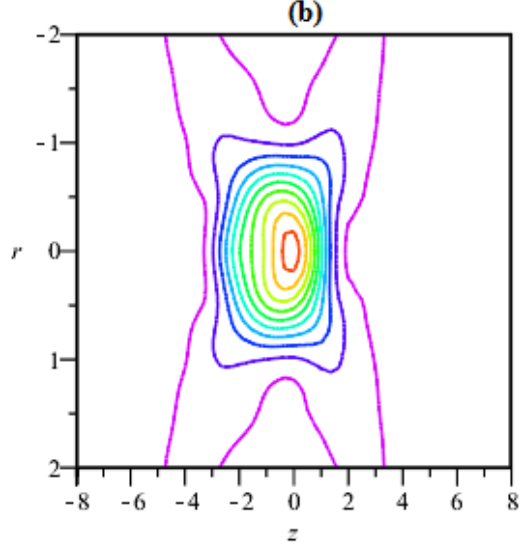

(c)

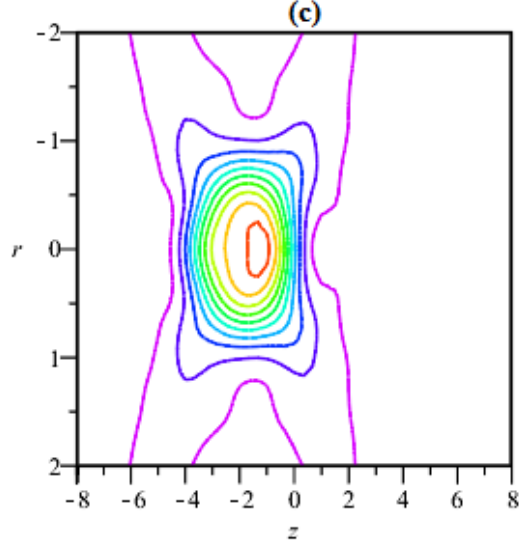

Fig.7. Normalized total intensity distributions in r-z plane incident R-TEM ${ }_{11} *$ beam under the condition of $\beta=1.08$, (a ) $C=0.25$, (b) $C=0.5$ (c ) $C=1$ respectively.

\section{CONCLUSION}

In conclusion, the focusing properties of the double ring shaped radially polarized multi Gaussian beam with radial cosine wavefront phase distribution are studied based on vector diffraction theory. The intensity distribution in the focal region is investigated in detail by numerical calculations for different cosine parameter values (C) and truncation parameter $(\beta)$. The simulation results show that the intensity distribution in focal region of the double ring shaped radially polarized multi gaussian beam can be adjusted considerably by the cosine parameter C. Focus can shift along optical axis on increasing $\mathrm{C}$, and focal pattern changes are observed. Moreover, by altering frequency parameter of the phase plate will change the energy distributions of maximum intensity peak and other small intensity peaks appeared and novel focal patterns also evolve considerably. The focusing properties technique may find wide applications in some domains such as optical tweezers, optical storage, particle manipulation, multifocal microscopy, laser printing.

\section{REFERENCES}

[1]. Davis, L. W., Patsakos, G, 1981. TM and TE Electromagnetic beams in free space, Opt. Lett. $6,22-23$.

[2]. Romea R. D., Kimura, W. D. 1990. Modeling of inverse Cherenkov laser acceleration with axicon laser-beam focusing, Phys. Rev.D 42, 1807-1818.

[3]. Bouchal,Z., Olivik,M., 1995. Non-diffractive vector Bessel beams,J. Mod. Opt. 42, 15551566.

[4]. Casperson, L.W., Hall, D.G., Tovar, A.A, 1997.Sinusoidal-Gaussian beams in complex optical systems, J. Opt. Soc. Am. A 14, 33413348.

[5]. Casperson, L.W. Tovar, A.A.1998. Hermitesinusoidal-Gaussian beams in complex optical systems, J. Opt. Soc. Am. A 15, 954-961.
[6]. Tovar, A.A., Casperson, L.W. 1998. Production and propagation of Hermite-sinusoidal Gaussian laser beams, J. Opt. Soc. Am. A 15, 2425-2432.

[7]. Lü,B., Zhang,B., Ma,H, 1999. Beam-propagation factor and mode-coherence coefficients of hyperbolic-cosine-Gaussian beams, Opt. Lett. 24, 640-642.

[8]. Lü, B., Ma,H., Zhang, B. 1999.Propagation properties of cosh-Gaussian beams, Opt.Commun. 164,165-170.

[9]. Eyyubo glu, H.T., Baykal, Y. 2005. Average intensity and spreading of cosh-Gaussian laser beams in the turbulent atmosphere, Appl. Opt. 44, 976-983.

[10]. Hricha,Z., Belafhal,A., 2005. Focusing properties and focal shift in hyperboliccosine-Gaussian beams, Opt. Commun. 253, 242-249.

[11]. Zhang, Y.C., Song, Y.J., Chen, Z.R., Ji, J.H., Shi, Z.X.,2007. Virtual sources for a cosh Gaussian beam, Opt. Lett. 32,292-294.

[12]. Jian-Nong,C., Qin-Feng,X.,Gang,W,2011. Tight focus of a radially polarized and amplitudemodulated annular multi-Gaussian beam, Chin. Phys. B 20,114211-114215.

[13]. Dorn,R., Quabis,S., Leuchs,G,2003. Sharper focus for a radially polarized light beam,Phys. Rev. Lett. 91, 233901

[14]. Yoon, Y. J., Kim, W. C., Park, N. C., Park, K. S., Park, Y. P. 2009. Feasibility study of the application of radially polarized illumination to solid immersion lens-based near-field optics, Opt. Lett. 34,1961-1963

[15]. Kozawa, Y., Sato,S,2007. Sharper focal spot formed by higher-order radially polarized laser beams, J. Opt. Soc.Am. A 24,1793-1798

[16]. Kozawa, Y., Hibi,T., Sato, A., Horanai,H., Kurihara,M., Hashimoto, H., Yokoyama, H.,Nemoto,T., Sato,S.2011. Lateral resolution enhancement of laser scanning microscopy by a higher-order radially polarized mode beam, Opt. Express 19, 15947-15954. 
[17]. Kozawa,Y., Sato,S, 2012. Focusing of higherorder radially polarized Laguerre-Gaussian beam, J. Opt. Soc. Am.A 29, 2439-2443.

[18]. Ashkin,A., Dziedzic, J.M. Bjorkholm, J.E. Chu, S.1986. Observation of a single beam gradient force optical trap for dielectric particles, Opt. Lett. 11,288-290.

[19]. Visscher, K., Brakenhoff, G.J. 1992. Theoretical study of optically induced forces on spherical particles in a single beam trap I: Rayleigh scatterers, Optik 89, 174-180.

[20]. Li,Y., Wolf,E.1981. Focal shift in diffracted converging spherical waves, Opt. Commun.39, 211-215.

[21]. Chowdhury, D.R. Bhattacharya, K. Chakroborty, A.K. Ghosh, R. 2003. Possibility of an optical focal shift with polarization masks, Appl. Opt. 42, 3819-3820.

[22]. Stamnes, J.J. Spjelkavik, B. 1981. Focusing at small angular aperture in Debye and Kirchhoff approximations, Opt. Commun. 40, 81-90.

[23]. Givens, M.P. 1982.Focal shift in diffracted converging spherical waves, Opt. Commun.42, 145-148.

[24]. Li,Y.1982. Dependence of the focal shift on Fresnel number and f number, J. Opt. Soc.Am. 72,770-774.
[25]. Martines-Corral,M., Climent,V.1996. Focal switch: a new effect in low-Fresnel number system, Appl. Opt. 35,24-30.

[26]. Li,Y,1997. Focal shift and focal switch in dualfocus system, J. Opt. Soc. Am. A 14,1297-1300.

[27]. Gao, X., Zhou,F., Xu,W., Gan,F,2005. Gradient force pattern, focal shift, and focal switch in an apodized optical system, Optik 116, 99106.

[28]. Odeja-Castaneda,J., Berriel-Valdos, L.R.1988. Arbitrarily high focal depth with finite apertures, Opt. Lett. 13,183-185.

[29]. Martines-Corral, M., Climent, V, 1996.Focal switch: a new effect in low-Fresnel number system, Appl. Opt. 35, 24-30.

[30]. Richards,B., Wolf,E, 1959. Electromagnetic diffraction in optical systems, II. Structure of the image field in an aplanatic system, Proc. R. Soc. Lond. A Math. Phys. Sci.253 (1274) 358379.

[31]. Youngworth, K.S. Brown, T.G, 2000, Focusing of high numerical aperture cylindrical vector beams, Opt. Express 7, 77-87.

[32]. Prabakaran, K., Rajesh, K.B., Pillai, T.V.S., Chandrasekaran R. 2013.Focal shift of radially polarized axis symmetric Bessel modulated Gaussian beam with radial variance phase plate, Optik 124, 5454-5457. 